\title{
Aprendizaje en las redes sociales: literacidades vernaculares y académicas en la producción digital de jóvenes escolares
}

\author{
Learning on Social Media: Vernacular Literacy and \\ Academic Literacy in the Digital Production of \\ School Students
}

\author{
Andrea Valdivia \\ Instituto de la Comunicación e Imagen, Universidad de Chile
}

\section{Resumen}

\begin{abstract}
Las redes sociales juegan un rol protagónico en la vida cotidiana juvenil, en especial aquellas donde la imagen es central. La producción y circulación de textos digitales multimodales ha aumentado significativamente. Este artículo aborda las potencialidades y complejidades de la producción digital en Instagram desde los aprendizajes y las literacidades. A partir de una investigación etnográfica en un liceo público de Santiago de Chile, se presenta el estudio de las prácticas digitales de ocho jóvenes. Los materiales analizados provienen de entrevistas con jóvenes, docentes y madres, observaciones etnográficas in situ y digitales, y piezas visuales producidas por los jóvenes. Los resultados muestran distintos aprendizajes, cuyo nivel de complejidad se asocia a la intensidad de la producción de textos y la orientación y motivación. Las literacidades vernaculares dan cuenta de un saber práctico donde el inglés, el hashtag, la imagen y el humor son significativos en la producción digital. Los hallazgos también muestran casos de producción que trascienden el uso personal con función expresiva, guiados por orientaciones estratégicas de comunicación, política y educación. Esto, gracias a la interacción de literacidades académicas y vernaculares. Se concluye con la identificación de vacíos en la alfabetización digital donde la escuela podría aportar.
\end{abstract}

Palabras clave: producción digital, redes sociales, aprendizaje, literacidad vernacular, literacidad académica

\footnotetext{
Correspondencia a:

Andrea Valdivia

Capitán Ignacio Carrera Pinto 1045, Ñuñoa, Santiago, Chile. andrea.valdivia@uchile.cl

(C) 2021 PEL, http://www.pensamientoeducativo.org - http://www.pel.cl 


\title{
Abstract
}

\begin{abstract}
Social media play a leading role in the daily lives of young people, especially such media where images are central. There has been a significant increase in the production and circulation of multimodal digital texts in current times. This article addresses the potential and complexity of digital production on Instagram from the perspective of learning and literacies. Based on ethnographic research in a public secondary school in Santiago, Chile, this paper presents the digital practices of eight young people. The materials analyzed come from interviews with young people, teachers, and mothers, in situ and digital ethnographic observations, and visual pieces produced by young people. The findings show different learning, the level of complexity of which is associated with the intensity of text production and the orientation and motivation. Vernacular literacies are related to practical knowledge, where English, hashtags, images, and humor are significant in digital production. The findings also show some cases of production that transcend personal use with an expressive function, guided by strategic orientations of communication, politics, and education. For the latter, the interaction of academic and vernacular literacies is crucial. Finally, the paper identifies certain gaps in digital literacy, where the school could contribute.
\end{abstract}

Keywords: digital production; social media; learning; vernacular literacy; academic literacy

Nuestra vida cotidiana está mediada por las tecnologías digitales, situación que se extremó a consecuencia de la pandemia COVID-19. Las medidas de control sanitario y restricción social han intensificado el uso y dependencia de las redes sociales para estar conectados al trabajo, los estudios y la vida social en general. Este fenómeno global ha implicado el incremento en el uso de aquellas plataformas que se sostienen en la imagen y el video (Kemp, 2020; Shum, 2020). Se podría plantear que vivimos la radicalización de las transformaciones culturales iniciadas hace más de una década, en especial en nuestras prácticas comunicativas y de aprendizaje.

La digitalización y la masificación de las redes sociales como escenarios de participación han venido de la mano de prácticas de literacidad vernacular que trascienden por primera vez los espacios locales e íntimos, convirtiéndolas en saberes y formas de hacer fundamentales para participar en sociedad (Barton, 2010; Barton $\&$ Lee, 2012). Estas prácticas de lectura y escritura se sostienen en la multimodalidad, con un rol central de la imagen como disparadora de sentidos (Jurgenson, 2019; van Dijck, 2008; Valdivia, 2021). De ahí el poder de la visualidad para las generaciones más jóvenes. Las literacidades vernaculares digitales que son parte de esa vida cotidiana juvenil tienden a ser contrapuestas con aquellas propias de la escuela. Sin embargo, tal como pretendo mostrar en este artículo, ambas interactúan, a veces de manera complementaria, en las prácticas digitales de las y los jóvenes escolares.

\section{Redes sociales en las prácticas juveniles de producción digital: el caso de Instagram}

Para gran parte de las generaciones jóvenes, la experiencia asociada a Internet se configura a partir de sus prácticas en redes sociales (We Are Social \& Hootsuite, 2021). Las aplicaciones más utilizadas comparten ciertas características: centralidad en la imagen, facilidad de uso y herramientas para la interacción y rápida producción de contenidos. Instagram es una de las redes más populares. Los jóvenes la valoran por sus recursos atractivos 
y sencillos para la expresión y documentación (Alhabash \& Ma, 2017). Esta red social ha contribuido a la masificación de la producción de contenido visual y audiovisual casero que no requiere de recursos tecnológicos especializados. A través de ella se puede registrar, editar y compartir de una sola vez.

Una alta producción de contenidos en plataformas digitales centradas en la imagen, como Instagram, demanda el dominio de lenguajes visuales y multimodales y de habilidades comunicativas de diverso tipo: lógicas de producción y curatoría de contenidos, conformación de audiencias (Barton, 2018; Buckingham, 2006; Mills, Stornaiuolo, Smith \& Pandya, 2018; Valdivia, 2021) y estrategias para la configuración de comunidades de afinidad (Gee \& Hayes, 2011). Todas las anteriores son capacidades que tienen como base procesos permanentes de aprendizaje.

\section{Aprendizaje, literacidad y alfabetización digital}

El aprendizaje supone cambios a partir de la adquisición, producción y apropiación de conocimientos y herramientas culturales (Vigotsky, 1978). Desde esta mirada sociocultural, mientras el lenguaje da vida a la experiencia humana por medio de la configuración de sentido y significado, el aprendizaje es aquello que nos permite estar y ser en sociedad. Aprendemos para vivir a lo largo de nuestra experiencia humana y más allá de las instituciones educativas. El término vidas de aprendizaje (learning lives) (Sefton-Green \& Erstad, 2017; Erstad, Gilje, Sefton-Green \& Arnseth, 2018) es interesante, pues porta una noción de proceso constante, abierto y multi-situado. Aprendemos a participar en diferentes espacios sociales, desarrollando habilidades y potenciando cambios en ellos, con implicancias para nuestra vida personal y social.

El aprendizaje desde esta perspectiva es una práctica cultural (Lave, 1996; Lave \& Wegner 1991; Rogoff, 2003) caracterizada por una acción sistemática y recurrente, una orientación hacia necesidades humanas y una normatividad que facilita la estabilidad de las acciones (Postill, 2010). Esto supone una serie de actividades rutinizadas que incluyen acciones, objetos y usos (la dimensión del hacer); un conjunto de saberes prácticos y significados asociados a lo que se está aprendiendo y sobre el aprendizaje mismo (las dimensiones del saber hacer y del sentido) y, por último, el aprendizaje como práctica posee determinadas orientaciones y motivaciones (la dimensión del para qué) (Valdivia, Brossi, Cabalin \& Pinto, 2019).

Si toda práctica supone la configuración de sentido y significado en relación con lo que se hace, el dominio de los lenguajes que median la práctica es clave. Es aquí donde la literacidad adquiere relevancia. Siguiendo los aportes de los Nuevos Estudios de Literacidad, el manejo de la lectura y escritura no solo se asocia al desarrollo y adquisición de una habilidad específica, sino que de múltiples literacidades producto de la participación en diversos escenarios e instituciones sociales (Gee, 2012). Con el término literacidad se releva la dimensión social de las prácticas de lectura y escritura y se enfatiza el valor situado de las capacidades que se desarrollan a partir de toda experiencia social mediada por textos (Cassany, 2016; Zavala, 2002). Reconocer diversas literacidades permite tomar distancia de la legitimación de una única vía para participar con propiedad, aquella sustentada en la alfabetización escrita. Esto permite, además, examinar la dimensión política e ideológica de las literacidades (Hernández, 2019), pues se hace cargo de los vínculos, a veces de complementariedad, otras de conflicto, entre literacidades y sus implicancias en la vida social.

Las literacidades en tanto prácticas sociales dan cuenta de patrones comunes de uso de la lectura y escritura en esferas y actividades particulares en las que desplegamos nuestro conocimiento cultural con diversas motivaciones. Tradicionalmente, se asumen literacidades específicas para distintas esferas de participación tales como escuela, familia y trabajo. Hoy en día la realidad parece ser más fluida, pues vemos cómo diversas literacidades están presentes en una misma esfera y cómo los límites entre esferas se desdibujan (Barton 2010; Barton \& Lee 2013; Gee \& Hayes, 2011). 


\section{Literacidades vernaculares y alfabetización en el mundo digital}

Las literacidades vernaculares son todas aquellas prácticas informales de lectura y escritura, sin reglas ni procedimientos evidentes, totalmente voluntarias y orientadas por propósitos personales. Como consecuencia, los aprendizajes asociados son de tipo práctico e informal (Barton \& Papen, 2010). Este tipo de prácticas se ha vinculado tradicionalmente con expresiones orales y textos escritos presentes en espacios privados y compartidos localmente.

Si una parte importante de la vida cotidiana que ocurre en las esferas digitales está centrada en el ocio y la interacción (Valdivia, 2021), las prácticas de lectura y escritura que allí ocurren pueden ser consideradas vernaculares. Estas literacidades, sin embargo, poseen particularidades que las diferencian de las prácticas tradicionales: una circulación masiva con un potencial de alcance global, la hibridez de géneros y la multimodalidad con centralidad en la imagen (Barton \& Lee, 2012).

Las literacidades digitales tienen una estrecha relación con las prácticas de lectura y escritura que ocurren en los medios de comunicación o en relación con ellos (Buckingham, 2006; Livingstone, 2004). La literatura en esta línea, además de caracterizar las prácticas cotidianas, señala una serie de habilidades y conocimientos necesarios para participar en las esferas digitales. Es acá donde resulta pertinente introducir la noción de alfabetización digital, pues implica la definición de qué capacidades y conocimientos son mejores para participar en dichas esferas, orientando su desarrollo. Tal como señalan Montes y López (2017) para la discusión sobre lenguaje disciplinar, mientras la literacidad da cuenta de las capacidades para participar en prácticas sociales semióticas, la alfabetización da cuenta de manera específica de procesos intencionados de desarrollo de aquellas habilidades de lectura y escritura que permiten una adecuada participación.

Buckingham (2006) propone cuatro dimensiones para organizar las habilidades y conocimientos que aseguran óptima participación en el espacio mediático/digital: representación, que supone la capacidad de interpretar y producir determinadas selecciones de la realidad; lenguajes, que considera tanto el dominio de la multimodalidad, las formas retóricas y estéticas, y la construcción textual de la comunicación interactiva; audiencia, en términos de reconocer las distintas posiciones que se asumen en la participación y, por último, la dimensión de la producción que, junto con aludir a las capacidades técnicas para la creación y circulación de textos, hace referencia también a la comprensión de las implicancias sociales, económicas, ideológicas y éticas en la producción. Ejemplos de lo último son los dilemas que hoy ofrecen las noticias falsas, los algoritmos que determinan los contenidos a los que accedemos, los límites de la privacidad y la datificación (Livingstone, Stoilova \& Nandagiri, 2019; Pangrazio \& Selwyn, 2019).

Por lo anterior, es fundamental abordar en la discusión sobre literacidad y alfabetización digital los aspectos éticos, económicos e ideológicos de nuestras prácticas digitales, además de las dimensiones técnicas y comunicativas (Buckingham, 2019; Gee \& Hayes, 2011; Gee, Hull \& Lankshear, 2018).

\section{Literacidades académicas y disciplinarias}

La escuela, la universidad y el trabajo han sido las esferas que por tradición se asocian a las prácticas de literacidad de mayor legitimación. Lenguaje académico, lenguaje escolar, literacidad avanzada, todos nombres con los que se conoce a los lenguajes presentes en la escuela y que se caracterizan por un registro formal predominantemente verbal. Su uso tiene la finalidad de promover la adquisición de conocimientos disciplinares, el desarrollo de habilidades cognitivas y sociales y la comprensión conceptual (Snow \& Uccelli, 2009). A diferencia de las literacidades vernaculares, las prácticas asociadas al lenguaje de la escuela suponen conocimientos pedagógicos para el diseño y producción de actividades que conduzcan los procesos de aprendizaje. 
Montes y López (2017) proponen una distinción entre literacidad disciplinar y literacidad académica. La primera hace alusión a las prácticas de lectura y escritura de alta especialización y específicas a dominios de conocimiento. Las literacidades académicas, por su parte, son prácticas más generales asociadas a ámbitos formales de enseñanza, aprendizaje, producción y aplicación de conocimiento. Estas últimas suponen el desarrollo de habilidades que permiten un posicionamiento imparcial y la producción de una voz de autoridad, el manejo de un vocabulario específico y de determinados géneros discursivos, y el despliegue de estrategias de razonamiento argumentativo (Snow \& Uccelli, 2009).

Como he señalado, las prácticas digitales podrían impulsar cruces entre esferas de participación y la concurrencia de literacidades vernaculares y académicas. En este artículo, a partir de hallazgos de una investigación etnográfica conducida con estudiantes secundarios de un establecimiento público de Santiago de Chile, analizo y discuto los aprendizajes presentes en la producción digital en Instagram y las formas en las que las literacidades interactúan para favorecer la participación de las y los jóvenes. Las preguntas que orientan este documento son: ¿Cómo son asociados los aprendizajes a las literacidades vernaculares digitales de jóvenes secundarios chilenos? ¿Cómo es la relación de las literacidades vernaculares y académicas en estos aprendizajes?

\section{Método}

La investigación etnográfica estudió aprendizajes y estéticas asociadas a las prácticas digitales de jóvenes escolares a partir de sus rutinas en cuatro escenarios de participación: escuela, hogar, esfera digital y grupos de pares. La identificación de estos escenarios siguió la propuesta de Livingstone y Sefton-Green (2016), y tuvo como propósito organizar el trabajo de campo y ver su relación a partir de la vida cotidiana de las y los jóvenes. En términos metodológicos, se siguieron los aportes de la etnografía educativa (Anderson Levitt, 2006) y la etnografía digital (Pink, Horst, Postill, Hjorth, Lewis \& Tacchi, 2016).

Para este artículo, me centro en el aprendizaje como objeto de estudio y en ocho casos de jóvenes participantes con distinto nivel de producción digital en Instagram. El análisis se enfoca en las rutinas desplegadas en la esfera digital y en el colegio, por ser las más relevantes para el problema aquí abordado.

El acceso al grupo de jóvenes fue a través del liceo, comunidad educativa con la que he realizado distintos proyectos de investigación y colaboración desde el año 2013. El establecimiento municipal está localizado en una comuna de clase media de la zona centro de Santiago de Chile y tiene una población promedio de 1400 estudiantes, provenientes de diversas comunas de la capital.

El trabajo de campo se realizó entre 2018 y 2019 y consideró varias estrategias y técnicas de investigación. En primer lugar, dos ciclos de observación etnográfica in situ. El primero ocurrió entre abril y agosto de 2018, y contempló el seguimiento a cada joven en sus rutinas diarias en la escuela, hogar y grupo de pares. El segundo ciclo se realizó en abril de 2019 y se situó en el liceo, exclusivamente. En segundo lugar, durante los dieciocho meses que duró el trabajo de campo, se desarrolló una observación digital tanto de las redes sociales de los jóvenes participantes como de cuentas de Instagram de estudiantes, organizaciones y docentes del liceo. También se realizaron entrevistas en profundidad con cada joven (dos sesiones) y entrevistas etnográficas con docentes, directivos y madres de los jóvenes. Además, se solicitó a cada participante la selección de publicaciones de Instagram para el posterior análisis.

Los materiales analizados son de distinta naturaleza: transcripciones de 30 entrevistas, piezas visuales digitales y reportes etnográficos con base en las notas de campo. Los reportes se elaboraron a partir de tres unidades de estudio: los jóvenes, la escuela e Instagram. La estrategia de análisis para las dos primeras unidades combinó el análisis etnográfico con la teoría fundamentada (Strauss \& Corbin, 2002), lo que permitió configurar de 
manera inferencial las rutinas en los diversos escenarios y la categorización y conceptualización de los significados, conocimientos y orientaciones en torno a aprendizajes y las literacidades. En el caso de la unidad de estudio asociada con Instagram, la estrategia analítica combinó el análisis categorial y dialógico para dar cuenta de posiciones y configuración de audiencias (Valdivia, 2017). Se usó el software NVivo 12 para la organización y categorización de toda la información.

La investigación aseguró el tratamiento ético con todos los participantes, jóvenes y adultos, mediante el consentimiento y asentimiento informado. Se aseguró el anonimato, por lo que todos los nombres de las y los jóvenes han sido cambiados, y se obtuvo la autorización explícita de cada joven para la circulación de los contenidos digitales seleccionados.

\section{Casos}

La selección de los ocho casos tuvo como propósito ejemplificar la diversidad de prácticas de producción digital que fueron observadas. Con fines de caracterización, he organizado a los jóvenes en tres grupos según la intensidad de su producción.

Tabla 1

Casos participantes y nivel de producción digital

\begin{tabular}{cccc} 
& \multicolumn{3}{c}{ Casos } \\
\cline { 2 - 4 } Nivel de producción & Edad & Curso \\
\cline { 2 - 4 } Producción esporádica & Esteban & 15 & $2^{\circ}$ \\
& Ramiro & 17 & $4^{\circ}$ \\
& Andre & 15 & $2^{\circ}$ \\
\multirow{2}{*}{ Producción activa } & Isabel & 17 & $4^{\circ}$ \\
& Claudia & 17 & $4^{\circ}$ \\
& Gustavo & 17 & $4^{\circ}$ \\
\hline \multirow{2}{*}{ Producción avanzada } & Ana & 18 & $3^{\circ}$ \\
& Mario & 15 & $2^{\circ}$ \\
\hline
\end{tabular}

Fuente: Elaboración propia.

Esteban y Ramiro son jóvenes con una producción digital esporádica en Instagram; ni la fotografía ni la publicación son actividades frecuentes en su rutina. Sí lo son el seguimiento de perfiles de amigos, amigas, artistas, organizaciones políticas, medios de comunicación alternativos, perfiles de memes y de sus cursos.

Andre, Isabel, Claudia y Gustavo son del perfil de usuarios más activo en Instagram. A las actividades observadas en el grupo anterior, estas jóvenes suman la publicación constante de stories y posteos de su vida cotidiana y de memes. Dos de ellas, Isabel y Claudia, han tenido más de un perfil en Instagram, el personal y uno secundario dedicado a sus aficiones: Justin Bieber y los Doramas. Gustavo, por su parte, es un activo usuario de Instagram; en su cuenta publica contenidos de su vida cotidiana con amigas y amigos, y reposteos de cuentas de medios de comunicación alternativos y organizaciones políticas, como DGS, la organización feminista estudiantil en la que participa. 
Ana y Mario son los casos de productores más avanzados. Ambos administran más de un perfil en Instagram y cada uno de ellos con curatorías claramente diferenciadas. En sus producciones, se aprecian criterios de comunicación digital y estéticos que sustentan la curatoría. Los perfiles no personales son considerados profesionales: uno dedicado a la fotografía de paisajes, en el caso de Mario, y el otro es la plataforma digital de DGS donde Ana es la responsable de la producción y gestión digital.

\section{Resultados}

\section{Aprendizajes juveniles en Instagram}

El día a día de las y los jóvenes transcurre con un celular a mano, al igual que el de la mayoría de nosotros. Conversar por WhatsApp, revisar Instagram, escuchar música en Spotify o YouTube son acciones que tienen rutinizadas y automatizadas y que suspenden solo para algunas clases o cuando no disponen de Internet. Ya en casa, la actividad principal es el consumo de contenidos visuales y audiovisuales. Los juegos en línea son otra de las actividades preferidas en los momentos de ocio en casa. La observación de sus rutinas rápidamente mostró que las esferas de participación -hogar, escuela, grupos de pares y entorno digital-conviven con límites difusos. La participación en la última esfera ha permitido y potenciado dichos cruces.

La producción y publicación de contenidos para estos jóvenes ocurre principalmente en la plataforma de Instagram y su frecuencia e intensidad dependerá del tipo de perfiles de usuarios que describí en la sección anterior. La diversidad y complejidad de las actividades, el grado de conocimiento y reflexividad sobre la práctica digital varía, al igual que los aprendizajes asociados.

Esteban y Ramiro usan Instagram para seguir contenidos de otros. Ambos producen y publican con escasa frecuencia y, cuando lo hacen, es para documentar algo especial o que les gusta. Por lo general, se trata de escenas de su vida diaria que se registran de manera "muy random".

Pensé 'ah caleta' ${ }^{1}$ que no subo algo' y me dieron ganas de subir algo. No es como que... ponte tú, yo tengo amigos que suben caleta todas las semanas, y yo como que no estoy ni ahí, es cuando me da la gana nomás (...) No tengo problema en subirlo, pero si tiene significado, no voy a subir una foto de algo porque se ve bonito nomás (Esteban).

No estoy subiendo todo el rato, subo cosas equis ${ }^{2}$, si algo me llama la atención o si es algo chistoso $^{3}$ lo subo, de repente canciones (...) Subo harto letras de canciones en historias (Ramiro).

La publicación en Instagram en ambos casos está asociada a fotos instantáneas en el feed (pantalla de inicio), o algunas stories (sección localizada en el ícono del perfil para publicaciones de 24 horas). Este tipo de uso no parece requerir de conocimientos o recursos específicos, como sí ocurre con las prácticas de jóvenes con mayor actividad de publicación. Las reflexiones sobre qué y cómo aprenden en relación con sus prácticas en redes sociales son acotadas en Esteban y Ramiro. Tal como señala el primero, definir las habilidades necesarias para usar redes sociales es algo complejo, pues las tiene naturalizadas.

Es que a mí igual me cuesta, porque como yo nací con esto y como desde chico tengo esto yo no sé qué habilidades tengo que desarrollar porque como ya tengo eso (Esteban).

1. Expresión chilena que, en este contexto, significa hace mucho tiempo (nota de la correctora).

2. Expresión que quiere decir "cosas específicas" (nota de la correctora).

3. Expresión chilena que quiere decir divertido (nota de la correctora). 
Las motivaciones para usar y participar en Instagram se concentran en la entretención, el estar en contacto con amistades e informados en relación con sus intereses en torno a la música y actividades políticas.

Aquellos casos con producción más activa y administración de más de un perfil en Instagram dan cuenta de aprendizajes diversos, en términos de uso de herramientas, estrategias y saberes. Claudia, Isabel y Andre tienen gran cantidad y variedad de publicaciones en el feed: fotografías propias, la mayoría de ellas mismas y con amigas y amigos, memes descargados o reposteados, fotografías de artistas o series favoritas, entre otros. La edición con filtros de Instagram es algo recurrente, al igual que stories de situaciones cotidianas, donde el colegio y la habitación son escenarios habituales. Andre, además de ese tipo de contenidos, comparte publicaciones con fotos de sus dibujos, todos inspirados en el animé japonés.

En las cuentas destinadas a aficiones (Justin Bieber y Dorama), Isabel y Claudia solo suben contenidos visuales descargados de otras cuentas o sitios web, y algunos con edición de texto e imagen por parte de las jóvenes. Claudia, en el último tiempo, ha ampliado el tipo de contenido temático de su cuenta dedicada al Dorama. En este perfil, varios de los post son memes como el de la figura 1.

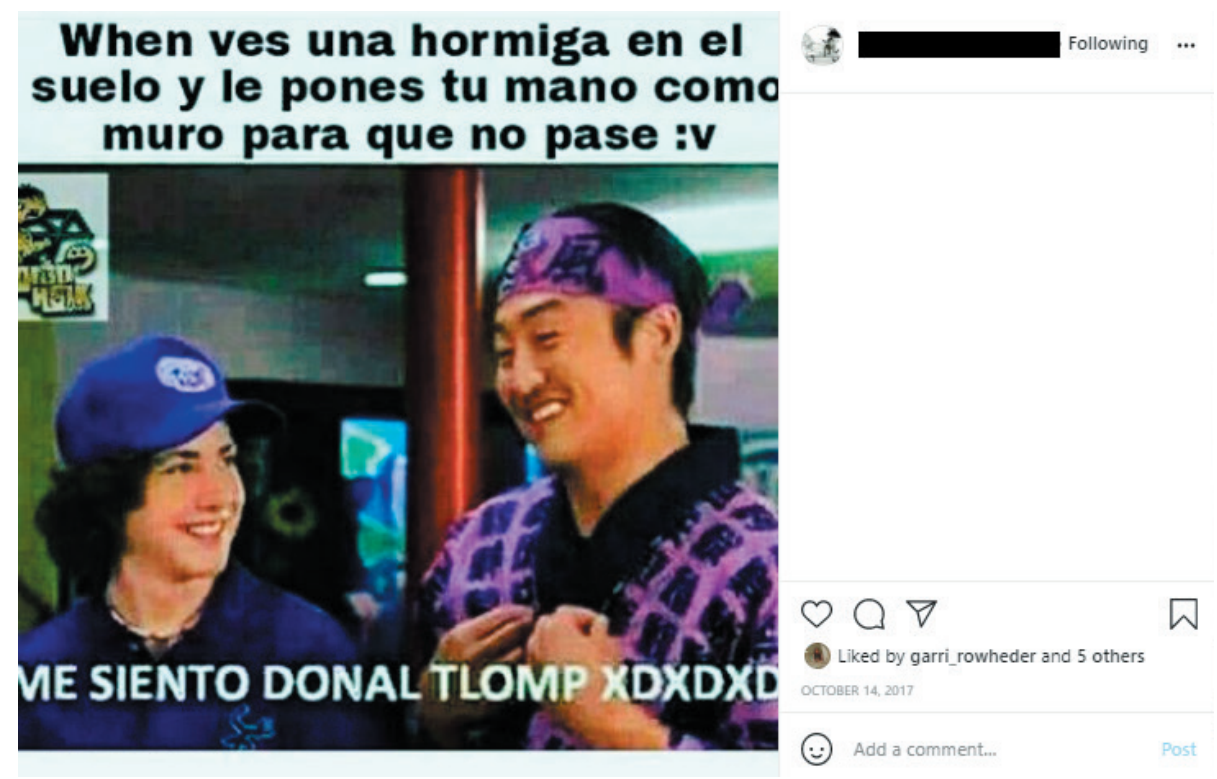

Figura 1. Meme publicado en Instagram Kdrama de Claudia (2017)

En esta publicación se conjugan varios aspectos clave para la participación y los aprendizajes juveniles en las redes sociales: la referencia a una cultura pop global, el humor y la inclusión del inglés en memes y piezas verbales.

El uso del hashtag para etiquetar, clasificar y lograr visibilidad de los contenidos publicados es una estrategia que Isabel utiliza, además del inglés, en varias de sus publicaciones. Dicha función no es algo que la joven explicite como conocimiento estratégico, tal como se aprecia a continuación:

Cuando empecé a seguir a Justin Bieber y viendo lo que hacían los famosos o viendo, no sé, mira se ocupa aquí el hashtag, ya vamos a usar hashtag, ya no sé, los emoticones, tus estados, ya vamos a usar emoticones (Isabel).

4. No se incluye la referencia exacta de la publicación, en esta figura ni en las siguientes, para respetar el anonimato de las y los participantes. 
Isabel reconoce la adopción de patrones de producción en redes sociales a partir de la imitación de estrategias de participación de gran popularidad. Hay un conocimiento implícito de lo que se supondría un desempeńo alineado con cierta norma. En este caso, la motivación del aprendizaje es estar alineada con la tendencia. Sin embargo, no es posible configurar conocimientos ni significados desarrollados de lo que implica participar produciendo contenidos y textos en Instagram.

Donde sí encontramos un conocimiento estratégico es en la cuenta profesional de Mario, espacio donde el joven comparte sus trabajos en fotografía digital de paisajes. El hashtag y el inglés son sus recursos más usados para captar la atención de seguidores y aumentar su audiencia. Para la elección de categorías a incluir en los hashtags, Mario recurre a buscadores de Internet que entregan un ranking diario de los tags más populares. La popularidad del hashtag puede ser más importante que la coherencia directa con la imagen. Por ejemplo, en la figura 2.i, de los 31 hashtags incluidos en el post los únicos en castellano son Chile y sin filtro. Varios corresponden a categorías asociadas con paisajes y naturaleza, aunque algunos no tienen que ver necesariamente con la imagen (ocean, beach, sunset, flowers, mountain, sea), a las estaciones del año, a fotografía y a calificaciones (amazing, awesome).
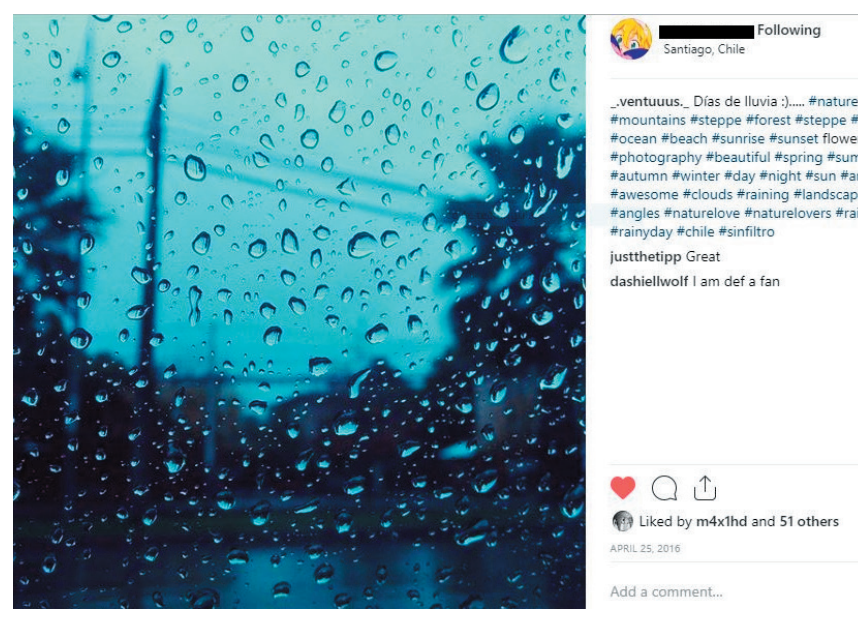

Publicación 22/04/2019

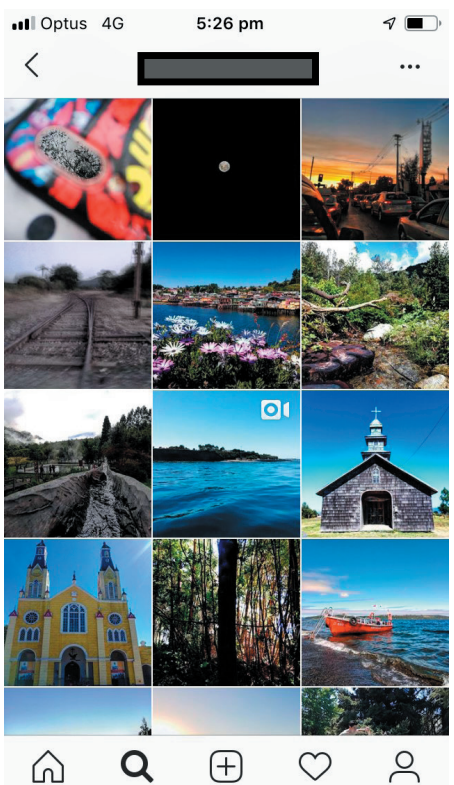

Pantalla de inicio, febrero 2019

Figura 2. Producción digital en Instagram profesional de Mario

Mario utiliza distintas herramientas de la comunicación y del marketing digital para lograr seguidores y un mayor alcance de este perfil. Además de los hashtags y del uso del inglés, el joven hace seguimiento de las estadísticas de visualizaciones, seguidores y likes que entrega la aplicación a las cuentas que son registradas como profesionales.

Este joven se define y es definido por su profesor jefe y madre como un fotógrafo. Dicha identificación se ha desarrollado a partir de su participación en el canal de televisión del liceo (iniciativa extracurricular coordinada por el profesor de artes visuales) y por la creación del perfil profesional en Instagram. Como dice Mario, "todo comenzó con el Instagram". Considerarse fotógrafo supone tener una noción estética y lenguaje visual que se manifiesta en la curatoría y creación fotográfica. El joven tempranamente definió la línea curatorial de su perfil: solo paisaje, fotografía instantánea y digital usando celular. Esto último resulta importante, Mario reitera varias veces el valor de la espontaneidad en la captura fotográfica. 
No es como que yo salga con la cámara porque un día quise sacar fotos, no. A lo que le saco fotos es a lo que yo veo y me gusta, lo que estoy viendo le saco foto, no es como que diga 'quiero una foto bien parecida a esta', sino que llego al lugar y si veo algo bonito agarro el mismo ángulo y saco la foto (Mario).

Espontaneidad en el momento de la captura fotográfica, pero experticia en el ojo fotográfico y en la edición. Mario juega y valora de sus fotografías los distintos encuadres, enfoques y la luz. En las fotografías de este perfil prima el color, el juego de planos y encuadres, y el uso de filtros, que está en varias de las publicaciones, tal como se aprecia en la figura 2.ii.

Una noción estética también se aprecia en las producciones de Ana, tanto en su perfil personal como en el administrado para DGS, la organización feminista en la que participa. En el primero, si bien no es evidente una línea curatorial estable, algunas de las publicaciones del feed son fotografías que le gustan especialmente, porque, como ella dice, "fueron fotos de días en que andaba inspirada". En la figura 3 se aprecia una de las fotografías que la joven seleccionó para mi análisis:

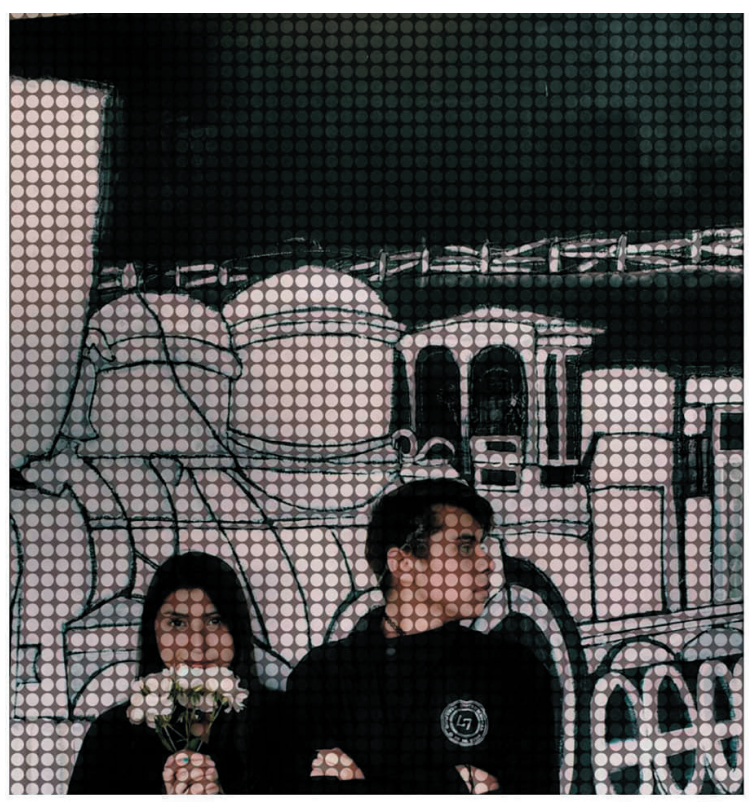

Figura 3. Publicación en el Instagram personal de Ana (2018)

La fotografía estaba acompañada del siguiente texto: "There's a crack in my window / A bird in my room / Angels all over", parte de la letra de una canción. Si bien este tipo de imagen no es común en el perfil personal de Ana, a ella le gusta porque en su producción hay un sentido de escenificación y porque la "edición quedó súper lograda". Estas marcas son muestras de su sentido artístico y estético, elementos que ella destaca como parte de su identidad. La edición que aplicó a la fotografía no recurre a los filtros que ofrece la aplicación de Instagram, ni son los más populares. Esto, sumado al diálogo que inicia con el extracto de la canción escrito en inglés, da cuenta de una publicación intencional que invita a una intimidad y sensibilidad compartida por una audiencia que sintoniza con esa estética.

Ana despliega con mayor énfasis su conocimiento estético y comunicacional en el perfil de DGS, la organización feminista en la que participa. La administración de esta cuenta implicó un salto tanto en el aprendizaje personal como en el de la organización. 
Nosotras empezamos a decidir esto, todo partió por lo que sacábamos en Instagram. [La primera publicación] era como 'Hola es el departamento', después fue como 'creo que estas pueden ser nuestras funciones'. Todo empezó como por Instagram y después se nos dio el tema de los testimonios [denuncias de acoso y abuso en el liceo] entonces dijimos como 'ok, tenemos que subir esto y la gente tiene que ver que queda plasmado'. Después se dio todo el tema del aborto, y con Zita nos acercamos a una coordinadora de la "8M" que conocimos por Instagram" (Ana).

Como se aprecia, Instagram es un escenario de aprendizaje, una fuente de información y conexión, y un precursor de aprendizajes que están fuera de él, aunque esto último no sea explícito en todos los casos. Lo que sí es evidente en todos es que la mayoría de los aprendizajes asociados con literacidades digitales ocurren en las redes sociales o a través de ellas.

“¿Cómo aprendí? Como he aprendido casi todo, metiendo mano”. Con esta cita de Mario se da cuenta del sentido del aprendizaje que estos jóvenes asocian al dominio y el saber hacer en redes sociales. Es un aprendizaje práctico, que conjuga la observación, la imitación, como contaba Isabel más arriba, pero también el "ensayo y error" y la indagación en la misma Internet o con sus pares.

[Y qué hiciste cuando no sabías, por ejemplo eso de cómo se etiqueta en Stories?]

Buscaba en Google o YouTube 'qué significa esto', 'cómo se usa' y ya, cuando no cacho 5 nada, ya ahí le pregunto a una amiga" (Andre).

El aprendizaje desde el hacer también está asociado con la exploración en experiencias de creación, como es el caso de Ana cuando cuenta cómo comenzó a fotografiar y editar para su perfil de Instagram:

¿Cómo fui aprendiendo? Yo creo que quería como canalizar mis sentimientos de la forma correcta.

Yo podía, un ejemplo básico, sentirme muy feliz y armar una foto de esa forma y luego intentar reflejar esa felicidad en mi creación y ok, y si me hace sentir esto y empezar a modificarla para crear al final lo que quiero demostrar y canalizar mis sentimientos (Ana).

Interés y necesidad están en la base del aprendizaje, en el caso de Ana, de crear, expresar y canalizar sus emociones. En todos, interés y necesidad de estar allí y de ser parte. La necesidad de producir textos no es algo transversal a las prácticas digitales juveniles, tal como vimos con Esteban y Ramiro, a quienes no los moviliza ese interés.

\section{El paso de la documentación y expresión personal a la creación de medios de comunicación escolares}

El celular y las redes sociales están en el colegio desde hace ya tiempo. Tal como dicen algunos docentes, tratar de prohibirlos es una batalla perdida, aunque algunos profesores lo hagan en sus clases. Instagram comenzó a dominar la escena de las redes sociales en el establecimiento el año 2016, cuando se popularizó y comenzó a ser el medio de comunicación usado entre jóvenes y por diversas organizaciones estudiantiles.

Durante el trabajo de campo de la investigación, los perfiles de Instagram llegaron a los cursos. Los más populares eran los Vendidas, perfiles sin administrador público donde se publican fotografías de estudiantes en poses o situaciones graciosas o poco favorecedoras. En palabras de Esteban "las Vendi'as son como las fotos cuando uno sale mal, uno dice 'ah la saliste vendiendo' por eso se llaman así los Instagram”. Las publicaciones en estas cuentas se concentran en el feed, lo que permite documentar y conservar el momento gracioso. La calidad de las fotografías no es algo que importe, sino la captura instantánea del momento y compartirlo para reírse del, o reírnos con, el compañero o compañera. Las reacciones en comentarios no son extensas y en su mayoría son con emoticones.

5. Expresión chilena que quiere decir entender (nota de la correctora). 
Estos perfiles son parte de la cotidianeidad de una clase, donde la broma y la burla han estado siempre presentes en las interacciones presenciales entre estudiantes. En los cursos que observé, las Vendidas parecían no ser fuente de conflicto ni de experiencias de vulneración por parte de las y los jóvenes, ni percibidas como tal por los docentes.

Lo que sí resultó ser fuente de preocupación y malestar para los adultos fueron los perfiles de organizaciones políticas, en especial, aquellos sin administrador identificado. Este establecimiento, al igual que varios de los liceos públicos del país, ha sido parte de los ciclos de movilización y protesta estudiantil iniciados el año 2006. En 2018, la acción política estuvo liderada por el movimiento feminista y tuvo como principal foco la denuncia de abuso y violencia de género al interior de los establecimientos educacionales. En esto, las redes sociales fueron clave para el accionar político juvenil.

Ellos [los estudiantes] se comunican por las redes sociales todo el rato, y creen que lo que sale en Instagram es ley. Ahí hay una distancia enorme entre la forma en la que nosotros vemos la realidad y cómo la ven ellos. Entonces todo se organizaba por ahí, los paros por Instagram, entonces nosotros como que quedamos descolocados, alguien decía una cuestión por Instagram, y listo, se rumoreaba y listo todos pensaban de esa forma, se instalaban como verdades (profesor de Historia).

Tal como manifiesta el profesor, hay una gran distancia entre adultos y jóvenes en sus maneras de ver la realidad y respecto de los criterios de veracidad en la información. Instagram como ley supone una legitimidad dada como fuente de información y como espacio para la toma de decisiones colectivas. Sin embargo, en lo que respecta a lo primero, los problemas de veracidad y desinformación asociados al consumo de contenidos políticosociales en redes fueron constatados tanto en jóvenes como adultos.

Instagram se convirtió en el principal medio de comunicación estudiantil, ya no solo para la interacción cotidiana y la participación digital, sino que para el acontecer de la comunidad en su conjunto. Como señalé más arriba, Ana asegura que la creación del perfil en Instagram fue clave para la conformación, instalación y visibilización de la organización feminista. En la presentación del perfil de Instagram de DGS (figura 4) se declara que su misión es "educar, mediar y concientizar" en relación con temáticas de discriminación sexual y de género. Tal como señala Gustavo, ellos estaban "contra toda desigualdad y abuso de poder".

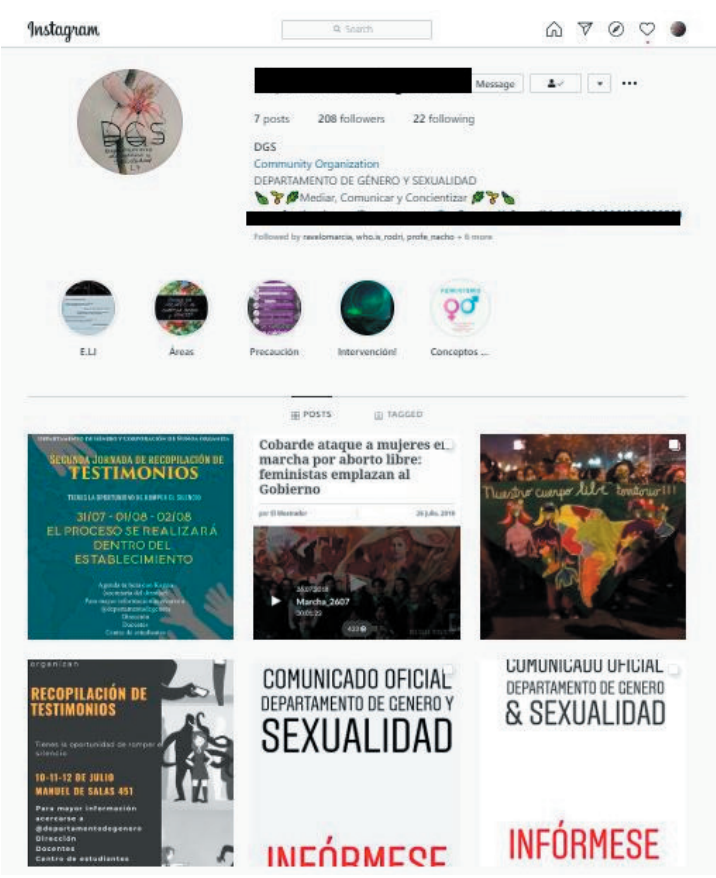

Figura 4. Pantalla de inicio del perfil Instagram del Departamento de Género y Sexualidades.

Fuente: Departamento de Género y Sexualidad (s.f.) 
Perfiles de Instagram como medios de comunicación con una función informativa es algo que se instaló con fuerza en el liceo en 2019. En ese año, aparecieron varias cuentas sin vínculo explícito a alguna organización o colectivo, todas con el propósito de informar sobre el acontecer y cotidianeidad del colegio y de cada curso: “i.p.e.17" (infórmate, piensa, expresa); "17_todoseptimos_"; "cosasdel7"; "17.informa”, son algunos ejemplos.

DGS puede ser visto como un precursor de dichos medios informativos; sin embargo, en esta plataforma se aprecia una apuesta más compleja. La organización no solo buscaba informar a la comunidad, sino también educarla y concientizarla en temáticas de género, sexualidad y feminismo.

Instagram fue la plataforma de posicionamiento y acción estratégica de DGS. Sus líderes coinciden en que la necesidad de generar contenido los impulsó a definirse como organización, a generar una suerte de manifiesto con sus objetivos y visión. En el post Comunicado oficial Departamento de Género y Sexualidades. Infórmese incluyen seis imágenes con un extenso documento PDF en donde está su declaración. En la primera publicación del feed, junto con la imagen del logo, se lee lo siguiente:

La función y objetivo principal es generar un ambiente de desarrollo óptimo para el estudiante, donde se fomente el respeto, la convivencia y el apoyo entre los diferentes integrantes de la comunidad escolar del liceo (...) esperando aportar a nivel nacional, con individuos conscientes e informados en los siguientes temas: Educación sexual, Sexo y género, La sexualidad desde los ojos de nuestros pares, Acoso y abuso sexual, Diversidad LGBT+, Cosificación y sexualización de la mujer en la sociedad, sexismo y educación No Sexista" (Departamento de Género y Sexualidad, 2018).

Llama la atención el uso de un registro académico con una voz impersonal para definir a la organización y su misión. Un discurso que resitúa a el/la estudiante, en tanto actor escolar, de su tradicional posición subordinada. El uso del lenguaje académico confiere cierta autoridad y legitimación a la misión educativa de DGS y, tal como veremos a continuación, será clave para la acción de esta organización.

\section{Articulación de literacidades vernaculares y académicas para la producción digital}

Trascender el uso de esta red social como fuente de entretención personal hacia este uso estratégico ha supuesto que los y las jóvenes amplíen sus repertorios de géneros y recursos discursivos. Esto es lo que se observa en la producción y curatoría del Instagram de DGS. Las publicaciones en este perfil no son abundantes, pero en cada una de ellas se observa especial cuidado en la visualidad y en los textos escritos que la acompañan. En estos últimos, el registro es formal y con una extensión que supera el común de publicaciones de los perfiles personales. Tal como vimos en el post Comunicado oficial, se trata de extensos documentos que presentan en detalle la organización, aclaran ciertos hechos y explicitan la posición y decisiones de la organización de manera argumentativa. Esto es coincidente con la intención política de sus líderes de posicionarse como referentes legítimos ante docentes y autoridades. Gustavo, al explicar el tono profesional de sus documentos -así descritos por Ana-, señaló que era la única forma de ser respetados y tomados en serio. Su propósito era incidir en el currículo escolar y convertir DGS en una unidad permanente en la institución.

En la visualidad y en la estrategia de producción digital de DGS también se aprecia un manejo de conocimientos y lenguajes disciplinares. Para Ana, como responsable de la creación de arte digital, la calidad de la imagen es clave para entregar un buen mensaje.

Para que la gente realmente entienda. Eso me gusta del trabajo de mi área, porque yo siempre he hablado de que las imágenes, los documentales y los videos siempre pueden llevarte a la emocionalidad y eso es clave para que la gente se enganche (Ana). 
Cuando Ana valora su trabajo y comenta los criterios usados para el diseño de afiches aparecen de manera explícita literacidades y conocimientos disciplinares de Artes visuales y Lenguaje y comunicación. La joven, mientras explica por qué eligió uno de los afiches digitales que fueron parte de la campaña de denuncias de acoso, señala:

Lo encuentro de alguna forma profesional, pero al mismo tiempo no cerrado, creo que yo nunca me voy tanto por los colores oscuros, al principio me fui por colores oscuros porque dije " $\mathrm{mmm}$ es un tema serio" (...) Es mi teoría del color que desarrollé en Tecnología, es que hice un trabajo sobre la teoría del color y de la forma, cómo se relacionaban entre ellos (Ana).

Es interesante cómo Ana y Mario, aquellos jóvenes con una producción más compleja y diversa en Instagram, no solo dan cuenta de aprendizajes y prácticas más elaboradas, sino que también enriquecen sus literacidades vernaculares digitales a partir de lenguajes y conocimientos académicos y disciplinares adquiridos y desarrollados en la escuela. En el caso de Mario, Artes visuales y, sobre todo, su participación en el canal de televisión, han sido la principal fuente de expansión de experiencias, conocimientos y repertorios visuales, que le han permitido desarrollar sus pasatiempos con experticia. Durante una de las sesiones de entrevista, hablando sobre sus referentes y fuentes de inspiración visual, Mario mencionó las producciones de Marvel. Para explicarme mejor qué de esas producciones le gustaban y valoraba, encendió su computador y buscó imágenes, durante largo rato describió y justificó la calidad visual de la fotografía seleccionada con una serie de conceptos y distinciones propias de un saber experto.

\section{Discusión y conclusiones}

Los resultados muestran que los aprendizajes asociados con la participación en redes sociales como Instagram varían según el tipo de uso y motivaciones que tengan los y las jóvenes, no así según frecuencia de uso de la red, pues todos sin excepción tienen un alto nivel de conexión a esta aplicación. Mientras más acotadas sean las actividades, más complejo será identificar saberes prácticos y significados en torno al hacer y al aprender. Esto ocurre en especial en casos de baja producción y circulación de contenidos, cuya motivación principal es estar conectado a amistades e intereses. Podría pensarse que el factor de producción de contenidos y textos multimodales en Instagram se asocia con aprendizajes más complejos; sin embargo, nuestros resultados no dan cuenta de esto. Es la dimensión de la orientación y motivación lo que marca una diferencia relevante entre los tipos de aprendizajes. Como vimos, la mayoría de los y las jóvenes tenían más de un perfil, pero solo aquellos que diferencian con claridad la administración de una cuenta personal de otra profesional o colectiva son los que poseen saberes prácticos y expertos. Estos casos, además, presentan orientaciones de comunicación estratégica donde la cuenta de Instagram se entiende como un medio o plataforma de un proyecto mayor.

Las literacidades vernaculares asociadas con estos aprendizajes confirman lo que señala Barton $(2010,2018)$ : la presencia de recursos y textos multimodales y visuales junto con el uso del inglés y el hashtag son clave para un sentido compartido, de pertenencia y con alcance global. Agregaría a esto el humor como recurso que motiva la participación y la lectura en todos los casos. El meme es uno de los contenidos más consumidos por estos jóvenes; como género discursivo se lee y se circula, pero no se produce, ni siquiera en los casos con una producción avanzada. El humor también está presente en la cuenta colectiva Vendi'as, donde los estudiantes trascienden el espacio de la sala de clases, registrando y conservando aquellos momentos que conforman el día a día de un grupo curso.

En el grupo de jóvenes con prácticas más complejas de producción digital apreciamos claramente la idea de que participar en una esfera social nos demanda aprendizajes y literacidades específicas (Barton \& Lee, 2013; Gee \& Hayes, 2011), pero a la vez potencia otros que van más allá dicho espacio. Tal como dicen Sefton-Green y Erstad (2017) se trata de procesos constantes, abiertos y multi-situados. Publicar fotografías profesionales o 
convertir un perfil de Instagram en un medio informativo, educativo o político impulsa a que se desarrollen conocimientos que no tienen que ver directamente con la administración de plataformas digitales; tal fue el caso de la organización feminista y de Ana, en particular. Es acá donde las esferas escuela y plataformas digitales mostraron su total porosidad, y donde las literacidades vernaculares y académicas se combinan y complementan. Vimos cómo los líderes de DGS recurren a lenguajes formales aprendidos en la escuela para legitimar su discurso, generando sus contenidos digitales en Instagram con léxicos formales y claves conceptuales, argumentativas propias del lenguaje académico (Snow \& Uccelli, 2009). También apreciamos cómo los saberes prácticos producto del aprender haciendo se sustentan en conocimientos de la visualidad y la comunicación aprendidos en el colegio.

La investigación nos muestra cómo las y los jóvenes convierten Instagram en una plataforma para la deliberación estudiantil, la información comunitaria y la educación entre pares, y cómo echan mano de los conocimientos y lenguajes disciplinares aprendidos en la escuela para asumir una posición de experto multimodal, y para disputar críticamente la autoridad escolar.

Por último, los aprendizajes de los jóvenes muestran el desarrollo en distinto grado de habilidades y conocimientos significativos para una alfabetización digital (Buckingham, 2006). Estas habilidades y conocimientos, eso sí, están concentradas en las dimensiones de representación y lenguaje. Aspectos propios de la dimensión de audiencia y producción con perspectiva crítica (Buckingham, 2019; Gee \& Hayes, 2011; Luke, 2012; Pangrazio, 2016) se aprecian escasamente. Este tipo de habilidades permiten no solo dominar lenguajes, sino que también comprender las redes sociales como plataformas comunicativas que son parte de lógicas éticas, económicas e ideológicas, donde el acceso y la circulación de textos ocurre mediado por algoritmos que guían la experiencia y participación (Kotilainen, Okkonen, Vuorio \& Leisti, 2020).

Parece ser que, tal como señalaba el profesor de Historia, "Instagram es ley” para los y las jóvenes, y desde allí no hay margen de duda o suspicacia respecto de la calidad y veracidad de los contenidos que pueden acceder, del diseño y arquitectura de la plataforma ni de las intenciones que hay detrás. Esta posición crítica estuvo ausente en las literacidades digitales del grupo de jóvenes, incluso de aquellos con una producción avanzada. Es aquí donde la escuela puede aportar promoviendo otros aprendizajes y otras formas de participar en la esfera digital, mediante una alfabetización digital crítica que, reconociendo la diversidad de aprendizajes juveniles, potencie la articulación entre literacidades vernaculares y académicas para una producción y participación reflexiva y clara de todas las complejidades de las redes sociales.

Financiamiento: este trabajo fue financiado con fondos del Programa Postdoctorado en el Extranjero de la Agencia Nacional de Investigación y Desarrollo de Chile, proyecto № 74180026.

Agradecimientos: la autora agradece a evaluadores y editoras del número especial por sus valiosos comentarios.

El artículo original fue recibido el 3 de marzo de 2021

El artículo revisado fue recibido el 14 de julio de 2021

El artículo fue aceptado el 22 de julio de 2021 


\section{Referencias}

Alhabash, S. \& Mengyan M. (2017). A Tale of Four Platforms: Motivations and Uses of Facebook, Twitter, Instagram, and Snapchat Among College Students? Social Media + Society 3(1). http://doi.org/10.1177/2056305117691544

Anderson Levitt, K. (2006). Ethnography. En G. Camilli, P. Elmore \& J. Green (Eds.), Handbook of Complementary Methods in Education Research (pp. 279-295). Washington, DC: American Educational Research Association/ Lawrence Erlbaum Associates, Inc.

Barton, D. (2010). Vernacular Writing on the Web. En D. Barton \& U. Papen (Eds.), The Anthropology of Writing. Understanding Textually-Mediated Worlds (pp. 109-25) Londres, Inglaterra: Continuum.

Barton, D. (2018). The Roles of Tagging in the Online Curation of Photographs. Discourse, Context \& Media, 22, 39-45. https://doi.org/10.1016/j.dcm.2017.06.001

Barton, D. \& Lee, C. (2012). Redefining Vernacular Literacies in the Age of Web 2.0. Applied Linguistics, 33(3), 282-298. https://doi.org/10.1093/applin/ams009

Barton, D. \& Lee, C. (2013). Language Online: Investigating Digital Texts and Practices. Londres, Inglaterra: Routledge.

Barton, D. \& Papen, U. (2010). The Anthropology of Writing. Understanding Textually-Mediated Worlds. Londres, Inglaterra: Continuum.

Buckingham, D. (2006). Defining digital literacy - What do young people need to know about digital media? Nordic Journal of Digital Literacy, 1(4), 263-277. https://doi.org/10.18261/ISSN1891-943X-2006-04-03

Buckingham, D. (2019). Teaching Media in a 'Post-Truth' Age: Fake News, Media Bias and the Challenge for Media/Digital Literacy Education, Culture and Education, 31(2), 213-231. https://doi.org/10.1080/11356405.2019.1603814

Cassany, D. (2016). Redes Sociales Para Leer y Escribir. En G. Bañales, M. Castelló \& N. A. Vega (Coords.), Enseñar a leer y escribir en la educación superior. Propuestas educativas basadas en la investigación (pp. 185-208). México: Universidad Autónoma de Tamaulipa.

Departamento de Género y Sexualidad [@departamentodegenero]. (s/f). Pantalla de inicio [Instagram]. Recuperado de https:/www.instagram.com/departamentodegenero/

Departamento de Género y Sexualidad [@departamentodegenero]. (2018, 17 de junio). El objetivo y función principal del Departamento de género y sexualidad es generar un ambiente de desarrollo optimo del estudiante, donde se fomente el respeto, convivencia y apoyo entre los diferentes integrantes de la comunidad escolar del liceo [Imagen de Instagram]. Recuperado de https://www.instagram.com/p/BkIqgCMhRlP/?utm_source=ig_web_copy_link

Erstad, O., Gilje, O., Sefton-Green, J. \& Arnseth, H. Ch. (2018). Learning identities, education and community. Cambridge, Inglaterra: Cambridge University Press.

Gee, J. P. (2012). Situated Language and Learning: A Critique of Traditional Schooling. Nueva York, NY: Routledge.

Gee, J. P. \& Hayes, E. (2011). Language and Learning in the Digital Age. Nueva York, NY: Routledge.

Gee, J. P., Hull, G. \& Lankshear, C. (2018). The New Work Order: Behind the Language of the New Capitalism. Nueva York, NY: Routledge.

Hernández, G. (2019). De los nuevos estudios de literacidad a las perspectivas decoloniales en la investigación sobre literacidad. Íkala, Revista de Lenguaje y Cultura, 24(2), 363-386. https://doi.org/10.17533/udea.ikala.v24n02a10

Jurgenson, N. (2019). The Social Photo. On Photography and Social Media. Nueva York, NY: Verso Books.

Kemp, S. (2020). Digital 2020: July Global Statshot. DataReportal-Global Digital Insights. Recuperado de https://datareportal.com/reports/digital-2020-july-global-statshot

Kotilainen, S., Okkonen, J., Vuorio, J. \& Leisti, K. (2020). Youth Media Education in the Age of Algorithm-Driven Social Media. En D. Frau-Meigs, S. Kotilainen, M. Pathak-Shelat, M. Hoechsmann \& S. R. Poyntz (Eds.), The Handbook of Media Education Research (pp. 131-139). Hoboken, NJ: John Wiley \& Sons, Ltd.

Livingstone, S. (2004). Media Literacy and the Challenge of New Information and Communication Technologies. The Communication Review, 7(1), 3-14. https://doi.org/10.1080/10714420490280152

Livingstone, S. \& Sefton Green, J. (2016). The Class. Living and Learning in the Digital Age. Nueva York, NY: NYU Press. 
Livingstone, S., Stoilova, M. \& Nandagiri, R. (2019). Children's Data and Privacy Online: Growing up in a Digital Age. An Evidence Review. Londres, Inglaterra: London School of Economics and Political Science.

Lave, J. (1996). La práctica del aprendizaje. En S. Chaiklin y J. Lave (Comp.), Estudiar las prácticas. Perspectivas sobre actividad y contexto (pp. 15 - 46). Buenos Aires, Argentina: Amorrortu editores.

Lave, J. \& Wenger, E. (1991). Situated learning: legitimate peripheral participation. Nueva York, NY: Cambridge University Press.

Luke, A. (2012). Critical Literacy: Foundational Notes. Theory Into Practice, 51(1) 4-11. https://doi.org/10.1080/00405841.2012.636324

Mills, K. A., Stornaiuolo, A., Smith, A. \& Pandya, J. (2018). Handbook of Writing, Literacies, and Education in Digital Cultures. Nueva York, NY: Taylor and Francis.

Montes, M. \& López, G. (2017). Literacidad y alfabetización disciplinar: enfoques teóricos y propuestas pedagógicas. Perfiles Educativos, 39(155), 162-178. https://doi.org/10.22201/iisue.24486167e.2017.155.58062

Pangrazio, L. \& Selwyn, N. (2019). 'Personal Data Literacies': A Critical Literacies Approach to Enhancing Understandings of Personal Digital Data. New Media \& Society, 21(2), 419-37. https://doi.org/10.1177/1461444818799523

Pangrazio, L. (2016). Reconceptualizing Critical Digital Literacy, Discourse: Studies in the Cultural Politics of Education 37(2)163-74. doi: 10.1080/01596306.2014.942836

Pink, S., Horst, H., Postill, J., Hjorth, L., Lewis, T. \& Tacchi, J. (2016). Digital ethnography. Principles and practices. Londres, Inglaterra: SAGE.

Postill, J.(2010). Introduction: Theorizing media and practice. En B. Bräuchler \& J. Postill (Eds.), Theorising media and practice (pp. 3 - 32). Oxford, Inglaterra: Berghahn.

Rogoff, B. (2003). The cultural nature of human development. Nueva York, NY: Oxford University Press.

Sefton-Green, J. \& Erstad, O. (2017). Researching "learning lives" - a new agenda for learning, media and technology, Learning, Media and Technology, 42(2), 246 - 250. https://doi.org/10.1080/17439884.2016.1170034

Shum, Y. M. (2020, julio 27). COVID-19 digital, internet, social media en el mundo. Yi Min Shum Xie. Recuperado de https://yiminshum.com/internet-digital-covid-19-2020/

Snow, C. \& Uccelli, P. (2009). The Challenge of Academic Language. En D. R. Olson \& N. Torrance (Eds.), The Cambridge Handbook of Literacy (pp. 112-33). Cambridge, Inglaterra: Cambridge University Press.

Strauss, A. \& Corbin, J. (2002). Bases de la investigación cualitativa: Técnicas y procedimientos para desarrollar la teoría fundamentada. Antioquia, Colombia: Editorial Universitaria de Antioquia.

Valdivia, A. (2017). What was out of the frame? A dialogic look at youth media production in a cultural diversity and educational context in Chile.Learning, Mediaand Technology, 42(1), 112-125.https://doi.org/10.1080/17439884.2016.1160926

Valdivia, A. (2021). Digital Production on Instagram: Vernacular Literacies and Challenges to Schools, Theory into Practice, 60(2), 171-182. https://doi.org/10.1080/00405841.2020.1857139

Valdivia, A., Brossi, L., Cabalin, C. \& Pinto, D. (2019). Alfabetizaciones y prácticas digitales desde agencias juveniles. Desafíos para la educación en Chile. Pensamiento Educativo. Revista de Investigación Educacional Latinoamericana, 56(2), 1-17. https://doi.org/10.7764/PEL.56.2.2019.1

van Dijck, J. (2008). Digital Photography: Communication, Identity and Memory. Visual Communication, 7(1), 57-76. https://doi.org/10.1177/1470357207084865

Vigotsky, L. S (1978). Pensamiento y lenguaje. Madrid, España: Paidós.

We Are Social \& Hootsuite. (2021). Digital 2021 Global Overview Report. We are Social. Recuperado de https://wearesocial.com/digital-2021

Zavala, V. (2002). (Des)Encuentros Con La Escritura. Escuela y Comunidad En Los Andes Peruanos. Lima, Perú: Red para el Desarrollo de las Ciencias Sociales en el Perú. 